\title{
Experimental study of defects influence on auxetic behavior of cellular structure with curvilinear elements
}

\author{
R. V. Goldstein, D. S. Lisovenko ${ }^{\dagger}$, A. V. Chentsov, S. Yu. Lavrentyev \\ †lisovenk@ipmnet.ru
}

Institute for Problems in Mechanics of RAS, prospect Vernadskogo, 101, b1, Moscow, 119526, Russia

\begin{abstract}
In this paper uniaxial tension of two-dimensional auxetic cellular structures is studied experimentally. Various samples were made by the laser cutting method from nonauxetic polyethylene terephthalate (PET-A amorphous) planes of size $112.5 \times 24 \times 0.7 \mathrm{~mm}$ with central area of $28 \times 24 \times 0.7 \mathrm{~mm}$. The transverse size of elements of hexagons was equal to sample thickness. The samples were subjected to monotonous uniaxial tension until the last moment when they still remained plane. Comparison of mechanical properties of the defect-free cellular structures consisting of concave hexagons with straight elements and concave hexagons in which part of straight elements is replaced with curvilinear elements is given as a result of experimental analysis. Cellular structure with straight elements showed lower value of Poisson's ratio in comparison with cellular structure with curvilinear elements. Influence of defects on mechanical properties of cellular structure with curvilinear elements is studied. We conclude that for the considered samples of cellular structure with curvilinear elements at uniaxial tension, loss of one horizontal element (near the center of the sample) changes effective mechanical properties much less than for the case of same structure with one vertical element being absent. As a result, tensile force - displacement diagram for four samples was calculated from the experimental data.
\end{abstract}

Keywords: auxetics, re-entrant honeycomb, defects, metamaterial.

\section{Introduction}

For isotropic materials Poisson's ratio varies in the range from -1 to 0.5 [1]. The majority of materials have positive values of Poisson's ratio. In the last decades rapid growth of number of theoretical and experimental studies on manufacturing and applications of materials and structures with negative values of Poisson's ratio is observed [2]. Such materials are now called auxetics $[3,4]$. To date, already more than four hundred crystal auxetics are known [5,6]. The greatest number (more than three hundred) belongs to cubic crystals [7]. Polymeric foams [8,9], composites [10-12], two-dimensional and three-dimensional cellular structures [13-15] are the examples showing auxetic properties.

The present paper reports the results of experimental study of two-dimensional auxetic cellular structures at tension. The concave hexagons with straight elements and concave hexagons in which a part of straight elements is replaced with curvilinear elements were chosen as a basis of the considered structures.

It is common that a concave hexagon with straight elements is used as a basic element of two-dimensional auxetic structures. In [17] we have introduced a hexagonal basic element in which a part of straight elements is replaced by curvilinear ones. In the present study we consider tension of two-dimensional auxetic structures with basic elements in the form of concave hexagons with straight elements and concave hexagons in which part of straight elements is replaced by curvilinear ones. Tensile behavior of similar samples containing structural defects was also considered.

\section{Methods}

The program of experimental studies included mechanical tests on uniaxial tension of a series of plane samples manufactured using femtosecond laser cutting method from a nonauxetic polyethylene terephthalate (PET-a amorphous) $0.7 \mathrm{~mm}$ thick plates. The laser machine was capable to perform displacements of the plate with micron accuracy, with the average power of the radiation of $3 \mathrm{~W}$. Duration of radiation impulses was $500 \mathrm{fs}$, energy of an impulse was $120 \mu \mathrm{J}$. Radiation power in one impulse reached $240 \mathrm{MW}$. Four plane samples of size $112.5 \times 24 \times 0.7 \mathrm{~mm}$ with central area of $28 \times 24 \times 0.7 \mathrm{~mm}$ were produced for this study (Fig. 1). The samples had the following design: sample $\mathrm{zz}-$ concave hexagons with rectilinear elements (Fig. 1a), sample ss concave hexagons with curvilinear elements (Fig. 1b), sample ssH - concave hexagons with curvilinear elements and one horizontal element removed (Fig. 1c), sample ssV concave hexagons with curvilinear elements and one vertical element removed (Fig. 1d). The design of samples featured the transverse size of structural elements to be equal to thickness of an initial plate.

All four samples were subjected to monotonous uniaxial tension on a MTS Synergie 400 setup at displacement rate of $1 \mathrm{~mm} / \mathrm{min}$ with simultaneous registration of displacements and forces. The samples were stretched until the last moment when they remained plane. Changes of sample geometry were registered in 12-Megapixel video and still images obtained during the experiment by Dahua IPC-HF81200E. Force and displacements of cantilever-moving beam of upper 
grip were also registered by the built-in sensors of the setup. Displacements and deformations were calculated after video acquisition and still images preparation using digital image correlation method in Ncorr software [18].

\section{Result and discussion}

The tensile force-displacement $\left(F-\Delta l_{y}\right)$ diagrams for four plane samples are given in Fig. 2a. Apparently from the figure, the diagrams of the studied samples stay similar up to a displacement value of $12 \mathrm{~mm}$. The maximum longitudinal displacement of sample $\mathrm{zz}$ is much less than the same for samples with curvilinear elements. Distinction is related with structure feature of curvilinear elements. The cellular structures containing curvilinear elements experience big plastic deformations. The photos for four considered samples at different stages of deformation are presented in Fig. 3b.

Variation of transverse displacement $\Delta l_{x}$ on longitudinal displacement $\Delta l_{y}$ for four samples is given in Fig. $2 \mathrm{~b}$. The sample zz attained smaller displacements in comparison with the samples containing curvilinear elements. The maximum change of longitudinal displacement for a sample with rectilinear elements was $9.6 \mathrm{~mm}$. For the case of samples with curvilinear elements maximum change of longitudinal displacement reached more than $18 \mathrm{~mm}$ (sample ss -
$18.6 \mathrm{~mm}$, sample ssH $-18.8 \mathrm{~mm}$, sample ssV $-20.8 \mathrm{~mm}$ ). Sample with one vertical element removed elongated much more than other samples containing curvilinear elements. It is related with the fact that due to absence of a horizontal element the sample lost it's stability later than other samples (see Fig. 3). Change of transverse displacement is also bigger for samples with curvilinear elements as compared to the sample zz. The maximum changes of transverse displacements were $6.4 \mathrm{~mm}$ for sample $\mathrm{zz}, 9.2 \mathrm{~mm}$ for sample ss, $8.9 \mathrm{~mm}$ for sample ssH and $7.2 \mathrm{~mm}$ for sample ssV.

Poisson's ratio in small deformations elasticity theory is defined by a formula $v=-\varepsilon_{x} / \varepsilon_{y}$, where $\varepsilon_{y}-$ longitudinal deformation, $\varepsilon_{x}$ - transverse deformation. By analogy with this formula in this paper we study variability of Poisson's ratio for samples with concave hexagons. In this case $\varepsilon_{y}=\Delta l_{y} / L_{0}$ longitudinal deformation, $L_{0}=28 \mathrm{~mm}$ - length of the central area, $\varepsilon_{x}=\Delta l_{x} / l_{0}-$ transverse deformation, $l_{0}=24 \mathrm{~mm}-$ width of the central area.

Calculation of Poisson's ratio dependence on longitudinal and transverse deformations for four samples is given in Fig. 4. Apparently from Fig. 4a the plane cellular structures containing curvilinear elements can experience increased longitudinal deformations. As a result of tension of twodimensional cellular structure with straight elements the maximum longitudinal deformations were obtained $40 \%$, and
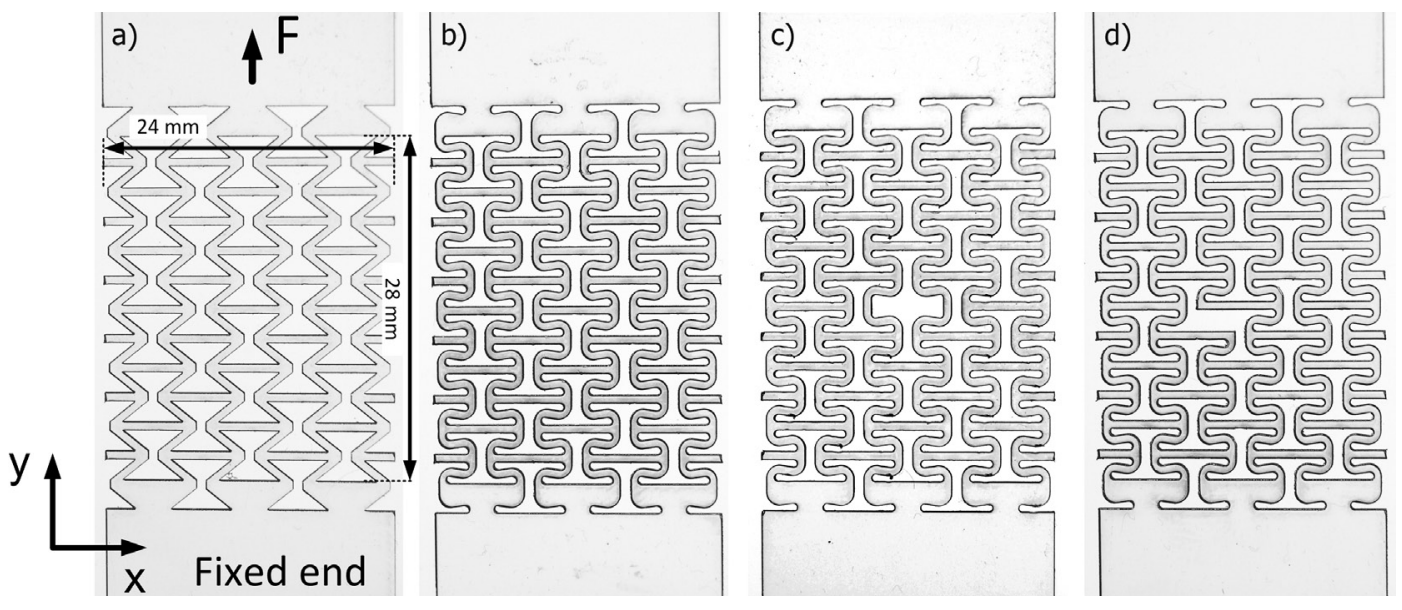

Fig. 1. Four plane samples with the central area $28 \times 24 \times 0.7 \mathrm{~mm}$ and the following designs: concave hexagons with rectilinear elements, sample $\mathrm{zz}$ (a); concave hexagons with curvilinear elements, sample ss (b); concave hexagons with curvilinear elements and one horizontal element removed, sample ssH (c); concave hexagons with curvilinear elements and one vertical element removed, sample ssV (d).
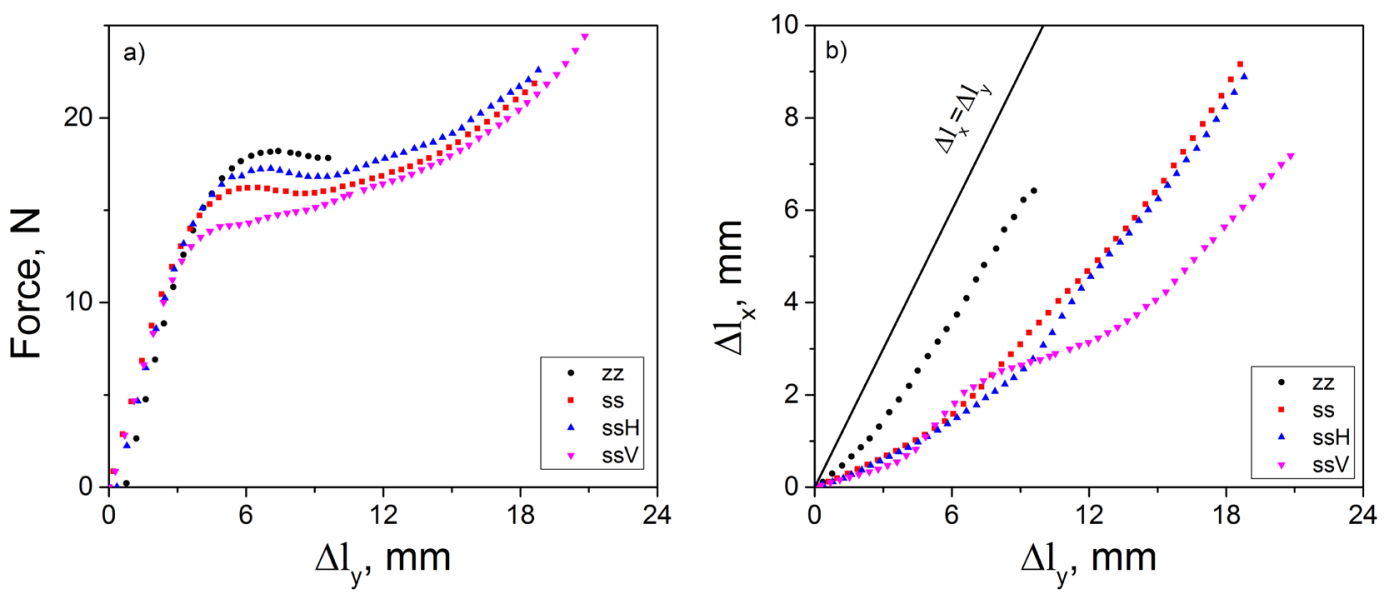

Fig. 2. Tensile force - displacement $\left(F-\Delta l_{y}\right)$ diagram (a) and variation of transverse displacement $\Delta l_{x}$ on longitudinal displacement $\Delta l_{y}$ (b). 
maximum transverse deformations reached $32 \%$. In case of samples with curvilinear elements the maximum longitudinal and maximum transverse deformations were $78 \%$ and $46 \%$ for sample ss, $80 \%$ and $44 \%$ for sample ssH, $88 \%$ and $36 \%$ for sample ssV. For sample ss and sample ssH longitudinal and transverse deformations have close values, i.e. it is possible to claim that lack of a horizontal element in cellular structure has weak influence on mechanical properties at an uniaxial tension. Lack of vertical defect (sample ssV) leads to stronger extension in comparison with sample ss and sample ssH (see Fig. 3).

Poisson's ratio for cellular structure with straight elements at small deformations is equal to -0.4 and then quickly decreases to -0.8 at increase of deformations. For samples ss and $\mathrm{ssH}$ the minimum measured value of Poisson's ratio were -0.58 and -0.55 respectively. It should be noted that for samples $\mathrm{zZ}$, ss and ssH, Poisson's ratio depending on longitudinal and transverse deformations monotonously decreases unlike the same for sample ssV were Poisson's ratio has complex nature of behavior. It should be noted that for sample ssV, lack of vertical element leads to lower transverse displacement and, therefore, lower transverse deformation as compared to sample ss and this is preserved in Poisson's ratio. The minimum value of Poisson's ratio for a sample ssV is -0.41 .
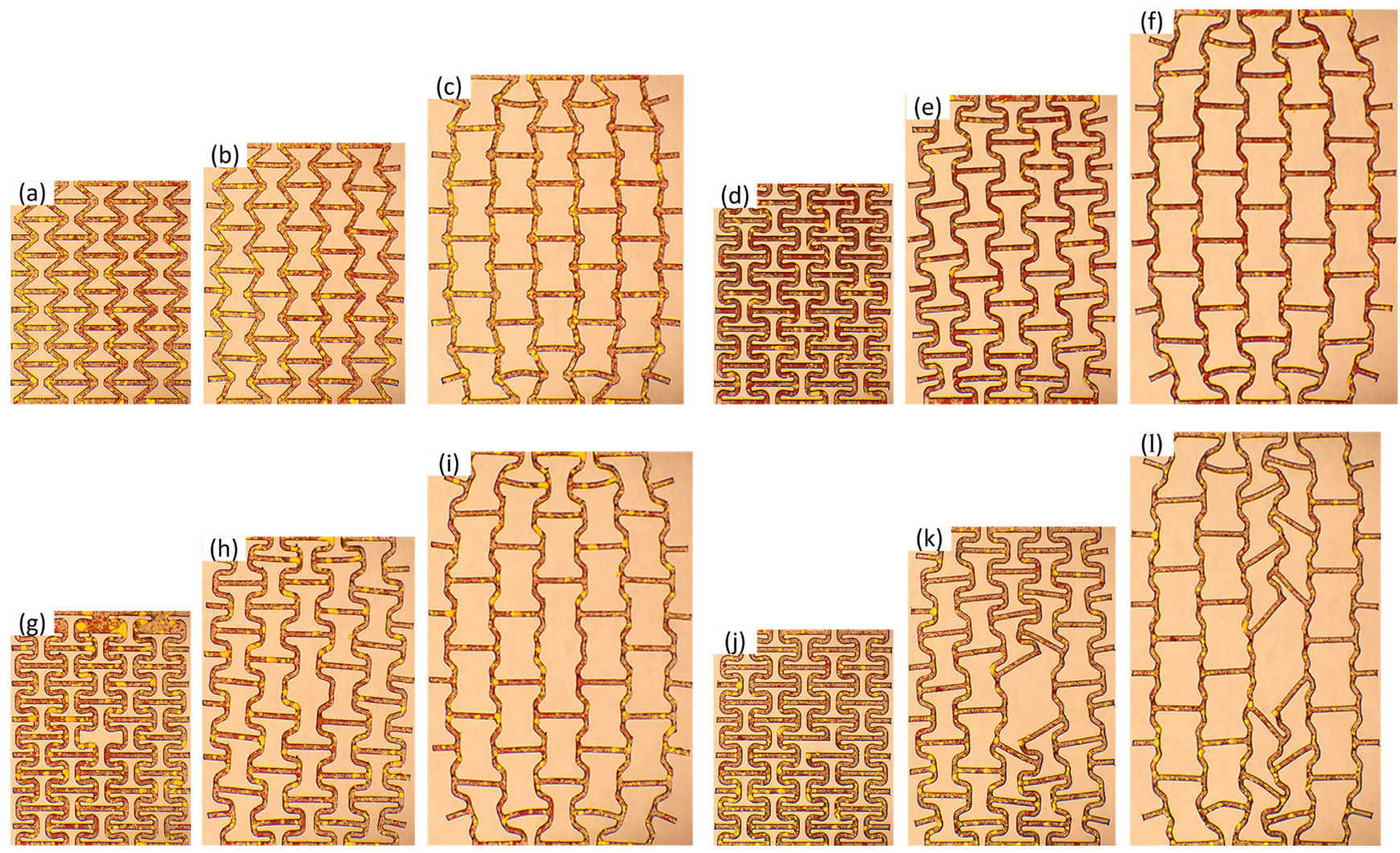

Fig. 3. Photographs of Sample zz at different stages of deformation $\varepsilon_{x}=0, \varepsilon_{y}=0$ (a), $\varepsilon_{x}=0.110, \varepsilon_{y}=0.172$ (b), $\varepsilon_{x}=0.319, \varepsilon_{y}=0.400$ (c); Sample ss at different stages of deformation $\varepsilon_{x}=0, \quad \varepsilon_{y}=0$ (d), $\varepsilon_{x}=0.167, \quad \varepsilon_{y}=0.396 \quad$ (e), $\varepsilon_{x}=0.457, \quad \varepsilon_{y}=0.785 \quad$ (f); Sample ssH at different stages of deformation $\varepsilon_{x}=0, \quad \varepsilon_{y}=0 \quad(\mathrm{~g}), \quad \varepsilon_{x}=0.139, \quad \varepsilon_{y}=0.470 \quad(\mathrm{~h}), \quad \varepsilon_{x}=0.443, \quad \varepsilon_{y}=0.800 \quad(\mathrm{i})$; Sample ssV at different stages of deformation $\varepsilon_{x}^{x}=0, \quad \varepsilon_{y}^{y}=0 \quad(\mathrm{j}), \quad \varepsilon_{x}^{x}=0.145, \quad \varepsilon_{y}^{y}=0.445 \quad(\mathrm{k}), \quad \varepsilon_{x}^{x}=0.360, \quad \varepsilon_{y}^{y}=0.881 \quad(\mathrm{l})$; Axis $y$ corresponds to tension direction, and axis $x$ corresponds to the transverse direction.
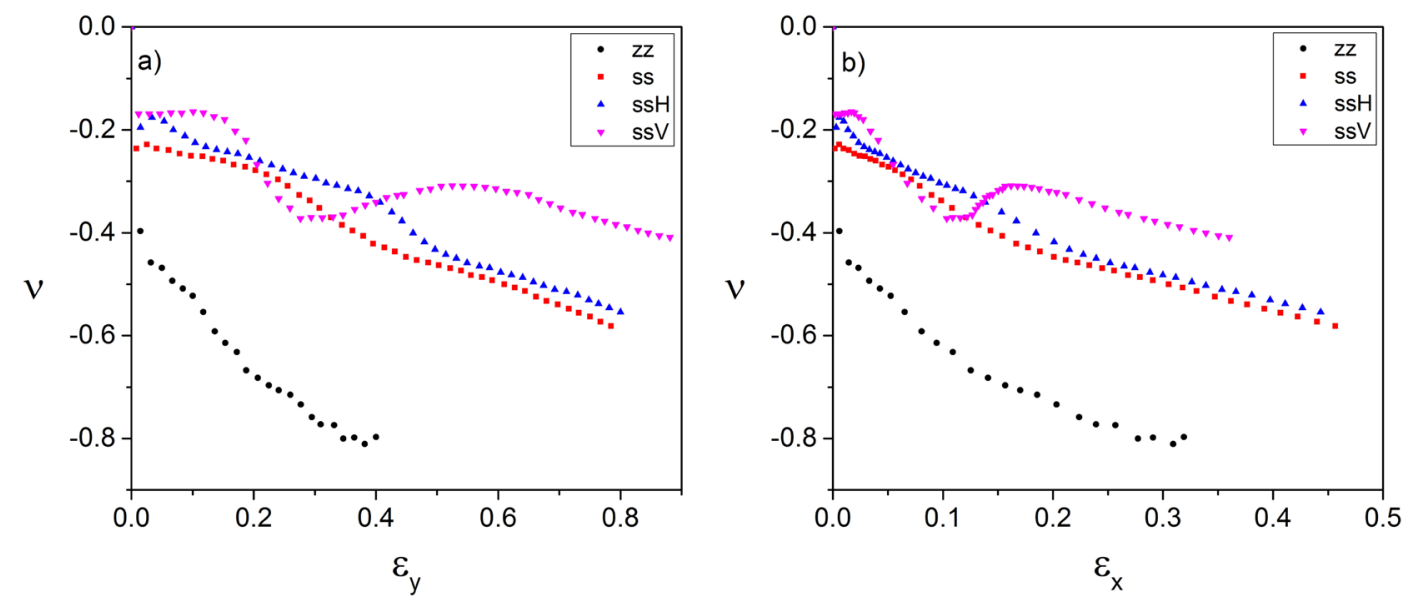

Fig. 4. (Color online) Poisson's ratio dependence on longitudinal (a) deformations $\varepsilon_{y}$ and (b) transverse deformations $\varepsilon_{x}$ for four samples. 


\section{Conclusion}

Mechanical properties of plane cellular structures with auxetic behavior at uniaxial tension are studied experimentally. Tensile tests of the sample consisting of rectilinear elements and faultless sample containing curvilinear elements showed that cellular structures with curvilinear elements extend stronger in comparison with structures with rectilinear elements. This is related to the fact that structures with curvilinear elements can experience stronger plastic deformations due to features of design. On the other hand cellular structures with rectilinear elements have higher negative values of Poisson's ratio. Experimental data analysis showed that lack of a horizontal element in cellular structure with curvilinear elements has little influence on its mechanical properties at uniaxial tension unlike the same for structure with removed vertical curvilinear element.

Acknowledgements. Authors are grateful to V. A. Gorodtsov and N. G. Soloviev for useful discussion of the results. The work was performed within the framework of the Project RFBR and Moscow Government N 15-31-70005 mol_a_mos.

\section{References}

1. L. D. Landau, E. M. Lifshitz. Theory of Elasticity. Vol. 7. Butterworth-Heinemann. (1986)

2. T.-C. Lim, Auxetic Materials and Structures. Springer Singapore. (2015) 588 p. DOI: 10.1007/978-981-287-275-3

3. K. E. Evans, M. A. Nkansah, I. J. Hutchinson, S. C. Rogers. Nature 353 (6340), 124 (1991). DOI: 10.1016/01609327(91)90123-S

4. K.E. Evans. Endeavour. New series 15 (4), 170 (1991). DOI: 10.1016/0160-9327(91)90123-S
5. Z.A.D. Lethbridge, R.I. Walton, A.S.H. Marmier, C. W. Smith, K. E. Evans. Acta Mater. 58 (19), 6444 (2010). DOI: 10.1016/j.actamat.2010.08.006

6. R.V. Goldstein, V.A. Gorodtsov, D.S. Lisovenko. Mech. Solids, 45 (4), 529 (2010). DOI: 10.3103/ S0025654410040047

7. R. V. Goldstein, V.A. Gorodtsov, D.S. Lisovenko. Phys. Status Solidi B, 250 (10), 2038 (2013). DOI: 10.1002/ pssb.201384233

8. R. Lakes. Science 235 (4792), 1038 (1987). DOI: 10.1126/ science.235.4792.1038

9. B. Brandel, R. S. Lakes. J. Mater. Sci. 36 (24), 5885 (2001). DOI: $10.1023 / A: 1012928726952$

10. K. L. Alderson, V.R. Simkins, V.L. Coenen, P.J. Davies, A. Alderson, K.E. Evans. Phys. Status Solidi B 242 (3), 509 (2005). DOI: 10.1002/pssb.200460371

11. T.-C. Lim. Phys. Status Solidi B 249 (7), 1366 (2012). DOI: 10.1002/pssb.201084220

12. M. Bilski, K. W. Wojciechowski. Phys. Status Solidi B 253 (7), 1318 (2016). DOI: 10.1002/pssb.201600140

13. R.V. Goldstein, V.A. Gorodtsov, D.S. Lisovenko. Eur. J. Mech. A Solids 63, 122 (2017). DOI: 10.1016/j. euromechsol.2017.01.001

14. R.F. Almgren. J. Elasticity 15 (4), 427 (1985). DOI: 10.1007/BF00042531

15. D. Li, L. Dong, R.S. Lakes. Mater. Lett., 164456 (2016). DOI: 10.1016/j.matlet.2015.11.037

16. D. Y. Fozdar, P. Soman, J. W. Lee, L.-H. Han, S. Chen, Adv. Func. Mater. 21 (14), 2712 (2011). DOI: 10.1002/ adfm.201002022

17. R.V. Goldstein, D.S. Lisovenko, A.V. Chentsov, S. Yu. Lavrentyev. Letters on Materials. 7 (2), 81-84 (2017). DOI: 10.22226/2410-3535-2017-2-81-84

18. J. Blaber, B. Adair, A. Antoniou. Exp. Mech. 55 (6), 1105 (2015). DOI: 10.1007/s11340-015-0009-1 